\title{
Examining the impact of transfers in pickup and delivery systems
}

\author{
Hiva Shiri ${ }^{a}$, Morteza Rahmani ${ }^{b^{*}}$ and Morteza Khakzar Bafruei ${ }^{a, b}$
}

\begin{tabular}{l}
${ }^{a}$ Industrial Engineering Department, T \\
${ }^{b}$ Industrial Engineering Department, \\
\hline C H R O N I C L E \\
\hline Article history: \\
Received June 7, 2019 \\
Received in revised format June \\
25,2019 \\
Accepted July 112019 \\
Available online \\
July 112019 \\
\hline Keywords: \\
Transfers \\
Pickup and delivery systems \\
Mixed integer programming
\end{tabular}

\section{Introduction}

Along with urban development, modern transportation systems are trying to reduce costs (transportation and road depreciation costs), reduce fuel consumption (cost and emissions reductions), and increase user satisfaction by optimizing usage of road infrastructure and vehicles capacities. Ridesharing (Lotfi et al., 2019), crowdsourced (Sampaio et al., 2018), mixed passengers and goods transportation (Godart et al., 2018), etc. are examples of modern pickup and delivery systems. The transfers capability (the load/passenger transfer between two vehicles in the middle of the route) is a relatively new feature, which in many cases, it can operationalize system in addition to reducing costs and increasing the efficiency of these systems. The pickup and delivery problem (PDP) is the generalization of the Vehicle routing problem (VRP), in which each request (load/passenger) must be taken from a specified location (origin) and delivered at a different one (destination). The problem objective is to determine the set of paths within the framework of several constraints so that the requests can be answered as best as possible. This objective is usually expressed as a combination of the vehicle cost (the service provider perspective) and the level of customer satisfaction (customer perspective). Express post service, postal couriers, shipping and carrier companies are the most major stakeholders

* Corresponding author

E-mail address: rahmanimr@yahoo.com (M. Rahmani)

(C) 2020 by the authors; licensee Growing Science. doi: $10.5267 /$ j.uscm.2019.7.003 
of PDP. The Pickup and delivery problem with transfers (PDPT) is the extension of PDP in which requests are allowed to transfer between vehicles in the given places (transfers points); the load/passenger transfer from one vehicle to another and continuing its route by the new one. By expanding solution space, transfers capability reduces costs throw optimal use of vehicle capacities, and increases the system flexibility in cases where it is impossible to meet demand without it. There could also be some constraints on the real system that require transfers. For example, it is only possible through transfers to limit the activity of each vehicle (or its driver) to a specific geographical area, while requests are widespread.

In the Shang and Cuff (1996) model, which firstly introduced PDPT, each network node is a transfer point. Subsequent research's in this area has been formed around mathematical modeling and problemsolving algorithms and techniques. Mues and Pickl (2005) provided a different integer programming model for the PDPT problem in integrated transport systems. Kerivin et al. (2008) modeled the PDPT problem with the split-delivery in the form of an integer programming model. A branch and bound algorithm was also developed, and random problem instances were solved with 5 to 15 requests. Rais et al. (2014) developed a new mixed integer mathematical programming model for the pickup and delivery problem with transfers. Thangiah et al. (2007) proposed a meta-heuristic algorithm for solving the PDPT under dynamic conditions with the split-delivery capability. In Gørtz et al. (2009), the authors considered the Dial-a-Ride Problem with transfers (DARPT). The transfers capability in a passenger transportation system can increase its overall productivity. In contrast, it could result in an increase in passenger dissatisfaction due to transfers operation and longer wait times. Hence, it is necessary to create a balance between the system flexibility and customer dissatisfaction which is the focus of research by Cortés et al. (2010). They proposed a mixed integer programming model. The Benders decomposition method was used to solve a small-scale problem, including six requests, two vehicles and one transfers point, and the results were compared with the results obtained from the branch and bound method.

Masson et al. (2011) used the Tabu search algorithm to solve the DARPT. Neighboring heuristic techniques are commonly used to solve routing problems with time-based constraints. Noting the dependence of routes in the PDPT, the time needed to determine the feasibility of a solution is one of the algorithm efficiencies factors. Masson et al. (2013b) proposed a method that allows the determination of the feasibility of a solution in constant time. In the Bouros et al. (2011) research, requests are randomly logged into the system and should be assigned to a fleet of vehicles. A two-step local search algorithm has been used to allocate requests to vehicles. Masson et al. (2013a) used the Large Neighborhood Search Method to solve the PDPT. According to their results, adding transfers points improves the objective function by $9 \%$ reduction. In a similar study, Masson et al. (2014) used the Large Neighborhood Search algorithm to solve the DARPT. Until now a fundamental question remains unanswered; what is and how much is the potential benefits of transfers capability? And what should be the structure and characteristics of the problem so that these benefits can be realized? The only research focused directly on this problem is Mitrović-Minić and Laporte (2006) who have partly answered these question (as noted by Sampaio et al. (2018)). A few researchers have also relatively responded to this question. Mitrović-Minić and Laporte (2006) is an empirical study on the usefulness of transfers in the pickup and delivery systems. To evaluate the benefits of transfers, they produced a sample of 50 and 100 requests in two uniform and clustering scenarios. They did not achieve satisfactory results from solving random samples with a different number of transfers points. So that, the addition of a transfer point in this sample does not significantly reduce the total distance traveled (objective function) relative to the without transfers point mode (between $0 \%$ and $7 \%$ on average for samples of 50 requests and between $2 \%$ and $10 \%$ in the samples of 100 requests). According to their results, the positive effect of transfers will increase by growth the size of the problem and the time window. The clustering problems sample results are very tangible (between 0 and $4 \%$, on average, depending on the cluster). These effects increase with increasing number of transfers points and depend on the cluster structure. The positive effect of the transfers is increased with shrinking the clusters. Nakao and Nagamochi (2008) examined the lower bound of traveling cost saved by adding a transfer 
point to the PDP. It is assumed that the number of transfers points is one, and each vehicle can visit a transfer point at most once. There is also no limitation on the number of vehicles. Vehicles have limited capacity, and the cost is asymmetric for each arc. The vehicle starts its journey from the origin and returns it, and all requests must be accomplished. Assuming that the $\mathrm{z}(\mathrm{PDP})$ is the optimal travel cost for PDP and $z(P D P T)$ is the optimal cost for PDPT; also, $p$ is the number of requests and $\mathrm{m}$ is the number of routes in the optimal solution of PDPT. They showed that following equations are valid:

$$
\begin{aligned}
& z(\mathrm{PDP})<(6[\sqrt{m}+1]) * \mathrm{z}(\mathrm{PDPT}) \\
& z(\mathrm{PDP})<(6[\sqrt{p}+1]) * \mathrm{z}(\mathrm{PDPT})
\end{aligned}
$$

which states that the travel cost saved by transfers can be proportional to the square root of the number of requests. Cortés et al. (2010) conducted research based on the need to evaluate the Dial-A-Ride system in two scenarios: with and without transfers. They emphasized the general mathematical modeling and the ability to find the optimal answer (or the near-optimal answer) as a strict way to compare the usefulness of methods (with and without transfers). In this study examining the conditions in which PDPT can produce a better optimal response than PDP has postponed to future, and the results are limited to the speculation that the usefulness of the transfers operation increases with the increase in demand. It has been proven in Qu and Bard (2012) that a necessary condition to reduce mileage along with the transfers in a PDPT, with a vehicle, is that the total customer demand be greater than the capacity of the vehicle. It is also proven that the transfers can be beneficial in the PDPT with two or more vehicles, although the capacity of the vehicle is not limited. Masson et al. (2013a) noted the clustering nature of requests in the sample problems of Li and Lim's (2003), and it is empirically demonstrated (based on experiment), given that the pickup and delivery points of the majority of requests are placed in a same cluster, the transfers cannot be so useful. Coltin and Veloso (2014) pointed out that transfers can have different effects depending on the objective function. For example, minimizing delivery times in proportion to minimizing costs can have more usefulness potential (more reduction in the objective function). Based on numerical results, Masson et al. (2014) concluded that the savings from the transfers, is very different from a sample of DARP to another, and it seems that the location and number of transfers points can have a negligible effect on it. According to their results, the reduction of the objective function (minimum cost) as a result of the trip, is close to zero on most problem samples (when the depot is the transfers place), and when all the point are transfers places, it varies from $0 \%$ to $10 \%$. They reported $1 \%$ to $9 \%$ cost reduction for real-life cases. Rais et al. (2014) stated that transfers capability could play a significant role in problems where travel distance and travel time available to vehicles, are limited. In such situations, requests can be moved between different vehicles at transfers points so as the limited routes or the maximum possible distances, can be bypassed. They also noted that their test data (Li \& Lim, 2003), are based on a Euclidean-based metric, that satisfies the triangular inequality, and does not create suitable conditions for the transfers. Also, the real-world networks may also have a much different cost structure and have a much more impressive use of transfers. The results of numerical tests in the study of Sampaio et al. (2018), showed that the introducing of the transfers capability in a crowdsourcing systems can significantly decrease the traveled mileage as well as the number of drivers required to complete a set of requests, especially when drivers have a short working time (relative to the planning horizon) and we are faced with longhaul requests. They analyzed the potential of transfers benefits in the urban pickup and delivery operations, with a particular focus on the conditions that drivers operate in short shifts (similar to crowdsourcing models). In this condition the flexibility, provided through the transfers, allows for the service the long-distance requests that otherwise would have been impossible. To investigate the potential for transfers capability, they produced a series of random samples and reported in both cases; with and without transfers, and reported a maximum reduction of $50 \%$ of the total distance and number of vehicles used. When the distance between pickup and delivery point is short, its usefulness is low and about $1 \%$ to $2 \%$, regardless of the length of the driver's shift. Also, the expected utility is reduced, 
with the increase in the length of the work shift; since with a longer shift, the driver can cover more distances and make more requests in the same route.

\section{Problem definition}

There is a fleet of vehicles with capacity, cost rates, and a specific origin and destination depot available for accomplishing a set of requests. Each request is a demand for the transfer of a load/passenger with a given volume/number from a pickup point (origin) to its delivery point (destination). Logically, each pickup or delivery node will only be visited by one vehicle; however, given the possibility of a transfers, any request can be reached by one or more vehicles from the origin to its destination. There is a set of predefined transfers points in the network and possibility of shifting the load between two vehicles at these points. At the end of the planning horizon, all vehicles must be in their destination depot, all requests will be accomplished and there will be no load at the transfers points. The goal is to complete all requests by obtaining the optimal value of the objective function (a combination of cost and customer satisfaction).

The most important assumptions of the problem can be summarized as follows:

- All information is already known.

- The fleet of vehicles is heterogeneous and has different capacity and cost rates.

- The origin and destination depots of the vehicles is given.

- The activity of each vehicle has a time window.

- Each request has its pickup and delivery point.

- Requests are inseparable, and each request must be shipped once.

- Each request has a time window for pickup and delivery action.

- Each request has a pickup and delivery service time.

- There is no inconsistency between requests, and each pair of requests can be carried out together, considering the capacity constraint.

- The set of transfers points (one or more) are predetermined, and the transfers operation is only possible at these points.

- Discharged load at transfers points can be temporarily stored throughout the planning horizon.

- The time and cost required for loading and unloading at transfers points are negligible.

- Any transfers point can service all vehicles simultaneously.

- Each vehicle can visit each transfers point at most once.

- The indefinite waiting for a vehicle is possible at pickup and delivery points up to the start of their time window, and it is possible to stop at the node until the time window is closed.

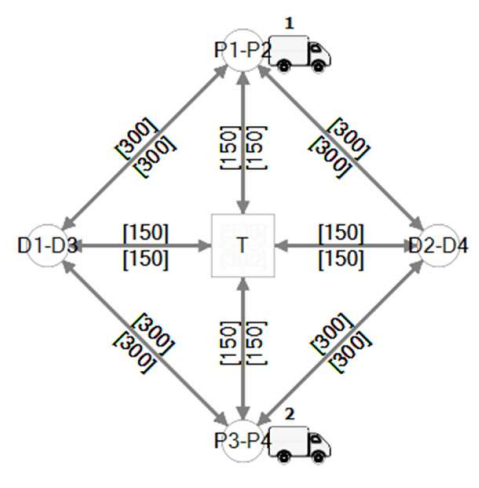

A. With transfers point

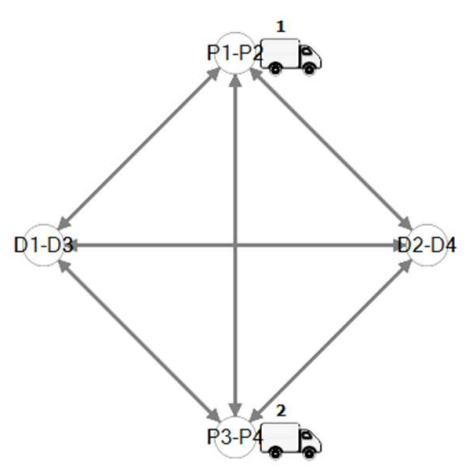

B. without transfers point

Fig. 1. Problem sample with two vehicles and four requests

To better understand, one problem sample is presented and solved in two scenarios; 1) without transfers, 2) with transfers. Fig. 1 (a) illustrates the problem model with the assumption of two vehicles and four requests (R1, R2, R3, R4) and with the possibility of transfers (Scenario 2). The request R1 should be 
moved from point $\mathrm{P} 1$ to $\mathrm{D} 1$, request $\mathrm{R} 2$ should be moved from point $\mathrm{P} 2$ to $\mathrm{D} 2$, and so on. The origin and destination depot of the vehicle 1 and the points $\mathrm{P} 1$ and $\mathrm{P} 2$, and the origin and destination depot of the vehicle 2 and the points P3 and P4 overlapped (placed in the same position). Also, the delivery points for requests R1 and R3, and requests R2 and R4, are overlapping. The distance between the nodes is noted on the arcs, and it is based on the Manhattan distance. The transfers point is marked with T. For the sake of simplicity, it is assumed that there are no time windows for requests and vehicles, and vehicles are completely identical and have no capacity constraint. Fig. 1b shows the same problem without the transfers.

Assuming that the cost of performing requests is equal to the total distance traveled by the vehicles, Fig. 2 is the solution to the problem without transfers. In this solution, only vehicle 1 is used and route traveled by this vehicle is as follows:

Vehicle 1: Depot-P1-P2-D2-D1-P3-P4-D4-D3-Depot

And its cost (mileage) is 1800 units. It should be noted that there are similar solutions at an equal cost for this scenario.

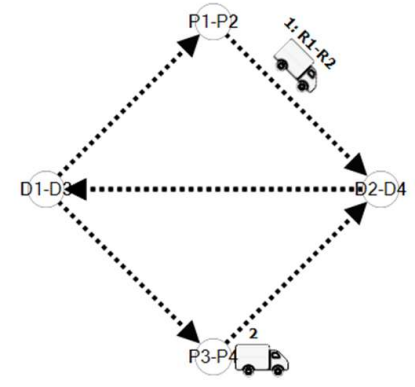

Fig. 2. Optimal solution without transfers

Fig. 3 shows the optimal solution to the problem with transfers. In this case, the route traveled by vehicles, is as follows:

Vehicle 1: Depot-P1-P2-T-D2-D4-Depot

Vehicle 2: Depot-P3-P4-T-D1-D3-Depot

And its cost is 1,200 units (600 units less than the first scenario). In the first step, vehicle 1 carries the loads R1 and R2, and vehicle 2 carries the loads R3 and R4 to the transfers point T (Figure. 3. A). At point T, R1 is moved from vehicle 1 to vehicle 2, and load R4 is transferred from vehicle 2 to vehicle 1. In the second step, the vehicle 1 with loads R1 and R4 and the vehicle 2 with the loads R1 and R3 leave the transfers point $\mathrm{T}$ and delivers the requests (Fig. 3. B).

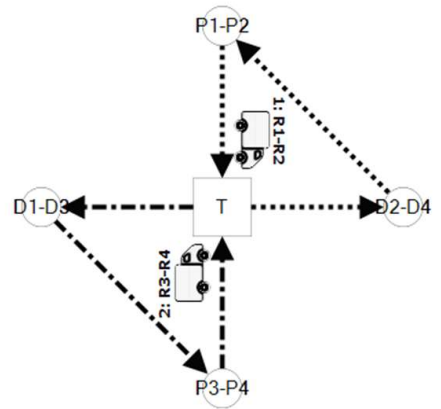

A. Vehicle 1 carries R1 and R2 and vehicle 2 carries R3 and R4 to the transfers point T.

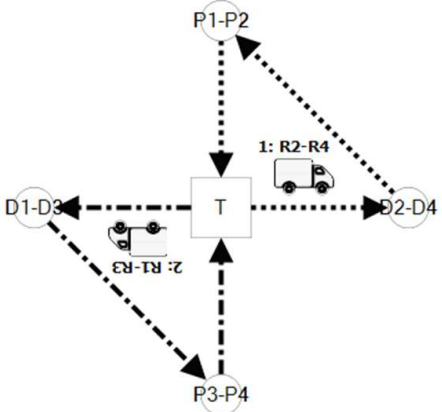

B. Vehicle 1 carries R2 and R4 and vehicle 2 leaves the transfers point $\mathrm{T}$ with $\mathrm{R} 1$ and $\mathrm{R} 3$

Fig. 3. Optimal solution with transfers 
Now, assuming that the time required to travel each arc is equal to its length, and the customer's satisfaction depends on reducing the wait time and riding time, the solutions of the two scenarios is considered from the customer's perspective (Table 1). Based on these results, in the first scenario, the average start time (wait time) and makespan for each request is 450 and 900 units, respectively. These values are 0 and 300 units for the second scenario, respectively. Hence transfers can increase customer satisfaction concurrent with decreasing costs.

Table 1

Optimal solutions from customer's perspective

\begin{tabular}{ccccccc}
\hline \multirow{2}{*}{ Request } & \multicolumn{2}{c}{ Without transfers (Scenario 1) } & \multicolumn{3}{c}{ With transfers (Scenario 2) } \\
\cline { 2 - 6 } & Wait Time & Ride Time & Total Time & Wait Time & Ride Time & Total Time \\
\hline R1 & 0 & 600 & 600 & 0 & 300 & 300 \\
R2 & 0 & 300 & 300 & 0 & 300 & 300 \\
R3 & 900 & 600 & 1500 & 0 & 300 & 300 \\
R4 & 900 & 300 & 1200 & 0 & 300 & 300 \\
\hline Average & 450 & 450 & 900 & 0 & 300 & 300 \\
\hline
\end{tabular}

\section{Mathematical modeling}

Assume that $G(N, A)$ is a directed graph with node-set $\mathrm{N}$ and $\operatorname{arc}-$ set $\mathrm{A}$. For each $i, j \in N$, the $\operatorname{arc}$ from $\mathrm{i}$ to $\mathrm{j}$ is defined as $i j \in A$. $\mathrm{V}$ is a heterogeneous vehicle set and indexed by $v=1, . .,|V|$. For each vehicle $\mathrm{v}$, its carrying capacity is denoted by $u_{v}$ and its origin and destination depots is denoted by $o(v) \in N$ and $\bar{o}(v) \in N$, respectively. $c_{i j}^{v}$ is the cost of traverse the arc $i j \in A$ by the vehicle $\mathrm{v}$. $\mathrm{R}$ is the customer requests set and is indexed by $r=1, . .,|R|$. The amount of the request $r$ or the required capacity is denoted by $q_{r}$. The pair $(p(r) \in N, d(r) \in N)$ is the pickup and delivery point of the request $\mathrm{r}$. For each request, a load with the size of $q_{r}$ should be transferred from $p(r)$ to $d(r)$. The set of transfers points is defined by $T \subseteq N$. The set $\mathrm{N}$ can be partitioned to the origin depots, destination depots, pickup, delivery and transfers points that are denoted with $O, \bar{O}, P, D, T$ respectively.

\subsection{Model}

The main idea of modeling and several constraints of the model are adopted from Rais et al. (2014).

$$
\begin{aligned}
& \text { Minimize } \quad \sum_{v \in V} \sum_{i j \in A} c_{i j}^{v} x_{i j}^{v} \\
& \sum_{j: i j \in A} x_{i j}^{v} \leq 1 \quad \forall v \in V, \quad i=o(v) \\
& \sum_{j: i j \in A} x_{i j}^{v}=\sum_{j: j k \in A} x_{j k}^{v} \quad \forall v \in V, \quad i=o(v), \quad k=\bar{o}(v) \\
& \sum_{j: i j \in A} x_{i j}^{v}-\sum_{j: j i \in A} x_{j i}^{v}=0 \quad \forall v \in V, \quad \forall i \in N-(O \cup \bar{O}) \\
& \sum_{v \in V} \sum_{j: i j \in A} y_{i j}^{r v}=1 \quad \forall r \in R, \quad i=p(r) \\
& \sum_{v \in V} \sum_{i: j i \in A} y_{j i}^{r v}=1 \quad \forall r \in R, \quad i=d(r) \\
& \sum_{j: i j \in A} y_{i j}^{r v}-\sum_{j: j i \in A} y_{j i}^{r v}=0 \quad \forall r \in R, \quad \forall i \in(P \cup D)-\{p(r), d(r)\} \\
& \sum_{v \in V} \sum_{j: i j \in A} y_{i j}^{r v}-\sum_{v \in V} \sum_{j: j i j \in A} y_{j i}^{r v}=0 \quad \forall r \in R, \quad \forall i \in T \\
& y_{i j}^{r} \leq x_{i j}^{v} \quad \forall i j \in A, \quad \forall r \in R, \quad \forall v \in V
\end{aligned}
$$




$$
\begin{aligned}
& \sum_{r \in R} q_{r} y_{i j}^{r v} \leq u_{v} x_{i j}^{v} \quad \forall i j \in A, \quad \forall v \in V \\
& t_{i}^{v} \geq a_{i}, \quad t_{i}^{v}+S_{i} \leq \bar{t}_{i}^{v} \leq b_{i}, \quad \forall i \in\{p(r), d(r)\}, \quad \forall v \in V \\
& t_{i}^{v} \leq \bar{t}_{i}^{v} \quad \forall v \in V, \quad \forall i \in O \cup \bar{O} \cup T \\
& \bar{t}_{i}^{v} \geq a^{v} \quad \forall v \in V, \quad i=O(v) \\
& t_{i}^{v} \leq b^{v} \quad \forall v \in V, \quad i=\bar{o}(v) \\
& \bar{t}_{i}+\tau_{i j}^{v}-t_{j} \leq M\left(1-x_{i j}^{v}\right) \quad \forall i j \in A, \quad \forall v \in V \\
& l_{i}^{r} \geq t_{i}^{v}-M\left(1-\sum_{j: j i \in A} y_{j i}^{r v}\right) \quad \forall r \in R, \quad \forall v \in V, \quad \forall i \in T \\
& \bar{l}_{i}^{r} \leq \bar{t}_{i}^{v}+M\left(1-\sum_{j: i j \in A} y_{i j}^{r v}\right) \quad \forall r \in R, \quad \forall v \in V, \quad \forall i \in T \\
& l_{i}^{r} \leq \bar{l}_{i}^{r} \quad \forall r \in R, \quad \forall i \in T \\
& x_{i j}^{v} \in\{0,1\} \quad \forall i j \in A, \quad \forall v \in V \\
& y_{i j}^{r v} \in\{0,1\} \quad \forall i j \in A, \quad \forall r \in R, \quad \forall v \in V \\
& t_{i}^{v}, \bar{t}_{i}^{v} \in \mathbb{R}^{+} \quad \forall i \in N, \quad \forall v \in V \\
& l_{i}^{r}, \bar{l}_{i}^{r} \in \mathbb{R}^{+} \quad \forall i \in T, \quad \forall r \in R
\end{aligned}
$$

The binary variable $x_{i j}^{v}$ is defined for each $i j \in A, v \in V$ to track the vehicle's route. If the vehicle $\mathrm{v}$ travers the arc $i j, x_{i j}^{v}$ is equal to one and zero otherwise. The constraint (2) means that each vehicle should only use one route to exit its origin depot. The sign $\leq$ shows that using all vehicles is unnecessary. According to the constraint (3), the vehicle that has moved, has to reach its destination and vice versa. The constraint (4) ensures the conservation of the vehicle's flow in the nodes. To track the movement path of each request, from the pickup to delivery point, the binary variable $y_{i j}^{r v}$ is defined for each $r \in R, i j \in A$ and $v \in V$. In case that the request $\mathrm{r}$ is carried by the vehicle $\mathrm{v}$ from the arc $i j$, $y_{i j}^{r v}$ is equal to one and zero otherwise. Constraints (5) and (6) will allow all requests to be picked up and delivered, respectively. Constraint (8) ensures request flow conservation at the transfers nodes and constraint (7) for other nodes. Constraint (9) creates a logical connection between shipping a load on an arc and movement of a vehicle on that arc. Constraint (10) indicates the vehicle capacity.

The two continuous variables $t_{i}^{v}$ and $\bar{t}_{i}^{v}$ are defined for modeling the arrival/departure time of the vehicle $v \in V$ to/from the node $i \in N$. Logically, $\overline{t_{i}} \geq t_{i}$ and the vehicle in the node i has $\overline{t_{i}}-t_{i}$ available time. Assume that $\left[a_{i}, b_{i}\right]$ is the time window of the node $i \in\{p(r), d(r)\}$ and $S_{i}$ is its service time. Constraints (11) and (12) connect the arrival and departure time of the node according to the time window and its service time. Assuming that $\left[a^{v}, b^{v}\right]$ is the time window of the vehicle $\mathrm{v}$, constraints (13) and (14) are established this. Also, $\tau_{i j}^{v}$ define the time needed to pass the arc $i j$ by vehicle $\mathrm{v}$, and for each arc $i j \in A$ that $x_{i j}^{v}=1$, the relation $t_{j} \geq \overline{t_{i}}+\tau_{i j}^{v}$ is established (constraint (15)).

A set of other logical constraints is required to establish the synchronization in the exchange of loads between the vehicles at the transfers points. For this purpose, two continuous auxiliary variables $l_{i}^{r}$ and $\bar{l}_{i}^{r}$ are defined as the time of arrival/departure of the request $\mathrm{r}$ from/to the transfers point $i$. The two constraints (16) and (17), set these variables. This happens when values are assigned to the load binary variables, defined on the input/output arc of the transfers node. Thus, spatial coordination is established 
as a prerequisite for timing coordination. The constraints (16) to (18) together cause the departure time of the outbound vehicle (carrying the $r$ load) exceeded the arrival time of the indoor vehicle (carrying the load r), to the transfers node, and the time synchronization occurs.

The default objective function of the problem is to minimize the cost of carrying out a series of activities using a vehicle fleet. Here, $c_{i j}^{v}$ is equivalent to the cost of passing the arc $i j$, by the vehicle v.

\subsection{Adding additional constraints}

Adding additional constraints to a model, derived from the properties and structure of a defined problem, can accelerate the solving process using the branch and bound algorithm. Two constraints are proposed to this end. The effectiveness of these constraints has been well proved by numerical tests.

$$
\begin{gathered}
\sum_{i: i d(r) \in A} y_{i d(r)}^{r v}+\sum_{j \in T} \sum_{i j \in A} y_{i j}^{r v} \geq \sum_{j: p(r) j \in A} y_{p(r) j}^{r v} \\
\forall r(p(r), d(r)) \in R, \quad \forall v \in V \\
\sum_{v^{\prime} \in V}\left(\sum_{i: u i \in A} y_{u i}^{r v^{\prime}}+\sum_{j: j d(r) \in A} y_{j d(r)}^{r v^{\prime}}\right) \geq \sum_{j: p(r) j \in A} y_{p(r) j}^{r v}+\sum_{i: i u \in A} y_{i u}^{r v} \\
\forall r(p(r), d(r)) \in R, \quad \forall v \in V, \quad \forall u \in T
\end{gathered}
$$

The constraint (23) states that if the request $r$ is picked up by a vehicle, it must be transferred by the same vehicle to the delivery node or transferred to one of the transfers nodes. The constraint (24) also states that if the request $r$ is picked up by a vehicle and moved to transfers node $u$, it should be carried out by one of the vehicles from this transfers node to its delivery node.

\section{Numerical results}

To investigate the effect of different parameters on the transfers benefits, several experiments designed and required sample problems generated. In this samples, the time horizon is 10,000 units, and the geographic scope of the requests are assumed to be a $1000 \times 1000$ square. Other parameters vary depending on the experiment. The model is coded in the GAMS environment, and the sample problems is implemented using a CPLEX solver on a PC with Dual-Core Pentium (R), $2.5 \mathrm{GHz}, 3 \mathrm{~GB}$ RAM, Windows $7(64 \mathrm{x})$ specifications. The big $\mathrm{M}$ is determined to equal 100,000 in numerical tests. The value of this parameter has a great influence on the execution time.

\subsection{Normal condition}

In the first experiment, it is assumed that all the parameters of the problem (vehicle capacity, time window, distance between the pickup and delivery of each request, etc.) are normal (not too big or too small). It is assumed that all requests, vehicles, and transfers points are distributed on a plane of $1000 \times 1000$ units. Three vehicles with carrying capacity of 10 units, the identical unit cost (equal to one unit), are placed in triangular scheme; three separate depots with coordinates $(250,285),(750,285)$ and $(500,715)$. There are 7 requests with random coordinates of $1000 \times 1000$ and length (direct distance between the pickup on and delivery points) between 200 to 1000 units, random quantity of 1 to 10 units, time window with a length of 2500 units and service time of 100 units. A transfer point is located at the center of three depots with coordinates $(500,500)$. Accordingly, 30 problem samples were generated and subjected to different tests. We called these instances "initial instances" throughout the rest of this paper. The system was considered in two scenarios in all experiments: 1- With transfers (PDPT), 2- Without transfers (PDP).

\subsubsection{Euclidean Distance versus Manhattan Distance}

It seems that the choice of Euclidean distance (direct distance), or Manhattan distance (which is obtained from the sum of the magnitudes of the difference in width and length), as the spatial and 
temporal metric, is the first parameter affecting the actual amount of transfers effectiveness. Obviously, the ground distances within the cities are mostly based on Manhattan distance, not Euclidean distance. To test this, "initial instances" solved once with Euclidean distance and once with the Manhattans distance assumption and the results are presented in Table 2. In this table, columns $\mathrm{z}, \mathrm{t}$ and $\mathrm{v}$ are the amounts of objective function (vehicles cost), execution time in seconds, and the number of used vehicles, respectively. Column "Gap (z)" displays the percentage change of objective function from without transfers scenario to with transfer scenario. According to the results of this table, in samples 10,25 , and 26, without using the transfers, the problem is infeasible. While assuming the transfers, the flexibility of the system has increased, and the problem becomes feasible. The cost reduction is in the range of $0 \%$ to $17.3 \%$ in Manhattan distance, and between $0 \%$ and $16.1 \%$ in Euclidean distance. The average cost reduction in Manhattan and Euclidean modes is $-5.7 \%$ and $-4.2 \%$, respectively, and shows that the reduction of costs is more tangible according to the Manhattan distance. In all subsequent experiments, Manhattan distance is used as metric.

In computing the averages in all the tables presented in this section, only rows are considered that have values in both models (PDP and PDPT). For example, to calculate the average number of used vehicles in the PDPT model in Table 2, the sample row of problems 10, 25, and 26, are not considered.

\subsubsection{The objective function}

Objective function in almost all of the research carried out on the transfers, is considered to be the cost of vehicles, while in the real world, we face different and more complex objective functions. In this regard, in order to measure the benefits of the transfers in different situations, "initial instances" with several different objective function including (1) total mileage, (2) the number of used vehicles and mileage, and (3) the total delay time, have been examined and compared (Table 3). The second objective function has two parts. First, the model minimizes the number of vehicles needed to handle requests, and in the second priority, reduces the cost of performing requests with this vehicle set.

According to the results of this study, while the transfers has reduced the average mileage cost by $5.7 \%$, in the second objective function, it is capable to reduce the number of used vehicles from 3 to 2 in the $43 \%$ of cases (the average value of used vehicles decreased from 2.6 to 2.1 ). At the same time, we have a 3.2 percent decrease in the cost (total distance).

Also, in the third scenario, the objective function (total delay time) decreased more than 100\% in $30 \%$ of cases, and the delay rate reaches zero in $13.3 \%$ of the cases. Also, the average delay rate has decreased from 571.5 to 247.5 . In terms of runtime, the second objective function needs much more time than the other two; 83.8, 1262.7 and 62.5 second for three objective functions, respectively.

\subsubsection{Scheme of the system}

The scheme of the pickup and delivery system with transfers; the way of placing the transfers points relative to the depots and the number of transfers points, is of great importance. Two experiments were conducted to measure the effect of this issue. In the first experiment (two transfers point), a transfers point with coordinates $(250,500)$ was added to "initial instances". It is expected by increasing the system capabilities, its flexibility and utility will also be increased. In the second experiment (Single point scheme), the scheme of "initial instances" changed and we have a central depot at $(500,500)$ with transfers capability. 
Table 2

Comparison effect of Euclidean and Manhattan metric on transfers

\begin{tabular}{|c|c|c|c|c|c|c|c|c|c|c|c|c|c|c|}
\hline \multirow{3}{*}{ Problem No } & \multicolumn{7}{|c|}{ Manhattan Distance } & \multicolumn{7}{|c|}{ Euclidean Distance } \\
\hline & \multicolumn{3}{|c|}{ PDP } & \multicolumn{3}{|c|}{ PDPT } & \multirow{2}{*}{$\operatorname{Gap}(z) \%$} & \multicolumn{3}{|c|}{ PDP } & \multicolumn{3}{|c|}{$\begin{array}{r}\text { PDPT } \\
\end{array}$} & \multirow{2}{*}{$\operatorname{Gap}(z) \%$} \\
\hline & $\mathbf{z}$ & $t(\sec )$ & $\mathbf{v}$ & $\mathbf{z}$ & t (sec) & $\mathbf{v}$ & & $\mathbf{z}$ & $t(\sec )$ & $\mathbf{v}$ & $\mathbf{z}$ & $\mathrm{t}(\mathrm{sec})$ & $\mathbf{v}$ & \\
\hline 1 & 6498 & 7.5 & 3 & 6498 & 15.7 & 3 & 0.0 & 5145.89 & 4.3 & 3 & 5145.89 & 9.7 & 3 & 0.0 \\
\hline 2 & 8160 & 13.2 & 3 & 7218 & 144.2 & 3 & -13.1 & 5724.05 & 9.4 & 2 & 5601.66 & 35.2 & 3 & -2.2 \\
\hline 3 & 6864 & 5.7 & 3 & 6796 & 10.7 & 2 & -1.0 & 5459.56 & 3.9 & 3 & 5450.36 & 6.4 & 2 & -0.2 \\
\hline 4 & 8734 & 13.1 & 3 & 8292 & 65.7 & 3 & -5.3 & 6737.63 & 8.9 & 3 & 6353.23 & 66.2 & 3 & -6.1 \\
\hline 5 & 8224 & 6 & 3 & 8224 & 24.1 & 3 & 0.0 & 6304.3 & 2.5 & 3 & 6227.92 & 11.2 & 3 & -1.2 \\
\hline 6 & 7964 & 6.1 & 3 & 7148 & 18.7 & 3 & -11.4 & 6044.73 & 2.7 & 3 & 5683.79 & 19.6 & 3 & -6.4 \\
\hline 7 & 5790 & 7.1 & 2 & 5790 & 10.1 & 2 & 0.0 & 4876.27 & 3.6 & 2 & 4876.27 & 12.6 & 2 & 0.0 \\
\hline 8 & 8386 & 11 & 3 & 7876 & 11.9 & 3 & -6.5 & 6367.24 & 6.4 & 2 & 6367.24 & 19.6 & 2 & 0.0 \\
\hline 9 & 7346 & 5.8 & 2 & 7156 & 8 & 3 & -2.7 & 5393.27 & 1.8 & 2 & 5393.27 & 3.3 & 2 & 0.0 \\
\hline 10 & - & - & - & 8748 & 11.1 & 3 & - & 7230.03 & 2.6 & 3 & 6806.37 & 14 & 3 & -6.2 \\
\hline 11 & 8764 & 9.1 & 3 & 8558 & 7.7 & 3 & -2.4 & 7082.07 & 1.9 & 3 & 7023.74 & 6.7 & 3 & -0.8 \\
\hline 12 & 5552 & 7.7 & 2 & 5552 & 29.8 & 2 & 0.0 & 4422.23 & 4 & 2 & 4422.23 & 17 & 2 & 0.0 \\
\hline 13 & 8540 & 5.5 & 3 & 7886 & 10.7 & 3 & -8.3 & 6534.91 & 1.9 & 3 & 6162.22 & 10.2 & 3 & -6.0 \\
\hline 14 & 6600 & 18.9 & 3 & 6222 & 192.6 & 3 & -6.1 & 5179.73 & 8.9 & 3 & 4964.01 & 177.8 & 2 & -4.3 \\
\hline 15 & 9380 & 9.8 & 3 & 7998 & 10.5 & 3 & -17.3 & 7503.02 & 6.4 & 3 & 6463.01 & 6.9 & 3 & -16.1 \\
\hline 16 & 6120 & 32.8 & 2 & 6106 & 1473 & 2 & -0.2 & 4946.58 & 63.9 & 2 & 4834.76 & 1637 & 2 & -2.3 \\
\hline 17 & 7164 & 13.8 & 3 & 6620 & 31.4 & 3 & -8.2 & 5597.25 & 10.7 & 3 & 4956.35 & 13.9 & 3 & -12.9 \\
\hline 18 & 7010 & 7.5 & 3 & 6346 & 12.7 & 3 & -10.5 & 5542.47 & 4.6 & 3 & 5171.37 & 14.6 & 3 & -7.2 \\
\hline 19 & 8508 & 9.4 & 3 & 7442 & 11.6 & 3 & -14.3 & 5900.39 & 4.4 & 3 & 5498.6 & 6.1 & 3 & -7.3 \\
\hline 20 & 8424 & 5.7 & 3 & 8288 & 7.2 & 3 & -1.6 & 6545.97 & 2.8 & 3 & 6373.22 & 7.1 & 3 & -2.7 \\
\hline 21 & 9072 & 5.5 & 3 & 8954 & 12.1 & 3 & -1.3 & 7284.98 & 1.9 & 3 & 7095.72 & 8.1 & 3 & -2.7 \\
\hline 22 & 7184 & 6.7 & 3 & 6530 & 10.3 & 3 & -10.0 & 5594.51 & 5.3 & 3 & 5245.67 & 14.3 & 3 & -6.7 \\
\hline 23 & 5506 & 5.3 & 3 & 5506 & 6.4 & 3 & 0.0 & 4418.8 & 1.9 & 3 & 4418.8 & 7.9 & 3 & 0.0 \\
\hline 24 & 7664 & 8.3 & 3 & 7116 & 7.1 & 3 & -7.7 & 5986.35 & 3.1 & 3 & 5665.3 & 5.7 & 3 & -5.7 \\
\hline 25 & - & - & - & 9692 & 6.5 & 3 & - & - & - & - & 7591.08 & 4.5 & 3 & - \\
\hline 26 & - & - & - & 7270 & 8.1 & 3 & - & - & - & - & 5970.23 & 4.9 & 3 & - \\
\hline 27 & 7562 & 7.3 & 3 & 7116 & 10 & 3 & -6.3 & 5863.97 & 3.8 & 3 & 5765.28 & 8.5 & 3 & -1.7 \\
\hline 28 & 8714 & 8.4 & 3 & 7902 & 28 & 3 & -10.3 & 6902.75 & 5.6 & 3 & 6038.31 & 14.8 & 2 & -14.3 \\
\hline 29 & 8802 & 7.2 & 3 & 8132 & 8.2 & 2 & -8.2 & 7069.6 & 1.5 & 3 & 6719.04 & 3.3 & 2 & -5.2 \\
\hline 30 & 7250 & 10 & 2 & 7250 & 85.2 & 2 & 0.0 & 6039.59 & 6 & 2 & 6039.59 & 233 & 2 & 0.0 \\
\hline Average & 7621.6 & 9.4 & 2.8 & 7204.5 & 83.8 & 2.8 & -5.7 & 5989.2 & 6.6 & 2.8 & $\mathbf{5 7 4 1 . 5}$ & 85.4 & 2.6 & -4.2 \\
\hline
\end{tabular}


Table 3

Comparing effect of different objective functions on transfers

\begin{tabular}{|c|c|c|c|c|c|c|c|c|c|c|c|c|c|c|c|c|}
\hline \multirow{3}{*}{$\begin{array}{c}\text { Objective function } \\
\text { Problem No }\end{array}$} & \multirow{3}{*}{$\frac{\text { Total distance }}{\text { Gap }(\mathrm{z})}$} & \multicolumn{8}{|c|}{ 1-Vehicle's count 2-Total distance } & \multicolumn{7}{|c|}{ Total delay } \\
\hline & & \multicolumn{3}{|c|}{ PDP } & \multicolumn{3}{|c|}{ PDPT } & \multirow[t]{2}{*}{$\operatorname{Gap}(z) \%$} & \multirow[t]{2}{*}{ Gap(v) } & \multicolumn{3}{|c|}{ PDP } & \multicolumn{3}{|c|}{ PDPT } & \multirow[t]{2}{*}{$\operatorname{Gap}(z) \%$} \\
\hline & & $\mathbf{z}$ & t (sec) & $\mathbf{v}$ & $\mathbf{z}$ & t (sec) & $\mathbf{v}$ & & & $\mathbf{Z}$ & t (sec) & $\mathbf{v}$ & $\mathbf{Z}$ & t (sec) & $\mathbf{v}$ & \\
\hline 1 & 0.0 & 7392 & 3.4 & 2 & 6944 & 479 & 2 & -6.5 & 0 & 0 & 5.3 & 3 & 0 & 6.1 & 3 & 0 \\
\hline 2 & -13.1 & 8160 & 42.5 & 3 & 7698 & 2074 & 2 & -6.0 & -1 & 2131 & 33.9 & 3 & 917 & 310 & 3 & $<-100$ \\
\hline 3 & -1.0 & 7312 & 5.5 & 2 & 6796 & 1429 & 2 & -7.6 & 0 & 171 & 4.3 & 3 & 171 & 44.5 & 3 & 0 \\
\hline 4 & -5.3 & 8734 & 123 & 3 & 8670 & 1000 & 3 & -0.7 & 0 & 601 & 36 & 3 & 6 & 230 & 3 & $<-100$ \\
\hline 5 & 0.0 & 8802 & 6.3 & 2 & 8694 & 388 & 2 & -1.2 & 0 & 113 & 3.8 & 3 & 39 & 20.6 & 3 & $<-100$ \\
\hline 6 & -11.4 & 7964 & 6.2 & 3 & 7868 & 192 & 2 & -1.2 & -1 & 128 & 2.9 & 3 & 0 & 4.2 & 3 & $<-100$ \\
\hline 7 & 0.0 & 5790 & 55 & 2 & 5790 & 4000 & 2 & 0.0 & 0 & 87 & 7 & 3 & 87 & 52.8 & 3 & 0 \\
\hline 8 & -6.5 & 9358 & 9.9 & 2 & 8350 & 169 & 2 & -12.1 & 0 & 187 & 5.7 & 3 & 187 & 75 & 3 & 0 \\
\hline 9 & -2.7 & 7346 & 4.8 & 2 & 7224 & 837 & 2 & -1.7 & 0 & 0 & 2 & 3 & 0 & 7.8 & 3 & 0 \\
\hline 10 & - & - & - & - & 8748 & 308 & 3 & - & - & - & - & - & 664 & 79.5 & 3 & - \\
\hline 11 & -2.4 & 8764 & 3.5 & 3 & 8666 & 40.7 & 2 & -1.1 & -1 & 1333 & 6.1 & 3 & 811 & 46 & 3 & -64.4 \\
\hline 12 & 0.0 & 5552 & 20.5 & 2 & 5552 & 4000 & 2 & 0.0 & 0 & 54 & 2.5 & 3 & 54 & 45 & 3 & 0 \\
\hline 13 & -8.3 & 8540 & 4.1 & 3 & 8818 & 150 & 2 & 3.2 & -1 & 1328 & 12.3 & 3 & 745 & 147 & 3 & -78.3 \\
\hline 14 & -6.1 & 6758 & 12.2 & 2 & 6500 & 4000 & 2 & -4.0 & 0 & 799 & 10.3 & 3 & 583 & 49.5 & 3 & -37.0 \\
\hline 15 & -17.3 & 9380 & 7.8 & 3 & 7998 & 484 & 3 & -17.3 & 0 & 901 & 7.4 & 3 & 0 & 6.6 & 3 & $<-100$ \\
\hline 16 & -0.2 & 6120 & 492 & 2 & - & 4000 & 2 & 0.0 & 0 & 0 & 6 & 3 & 0 & 37.4 & 3 & 0 \\
\hline 17 & -8.2 & 7574 & 75.4 & 2 & 7096 & 4000 & 2 & -6.7 & 0 & 786 & 8 & 3 & 719 & 162 & 3 & -9.3 \\
\hline 18 & -10.5 & 7010 & 7 & 3 & 6564 & 847 & 2 & -6.8 & -1 & 0 & 3.4 & 3 & 0 & 7.4 & 3 & 0 \\
\hline 19 & -14.3 & 8508 & 21.6 & 3 & 8766 & 1420 & 2 & 2.9 & -1 & 500 & 2.7 & 3 & 0 & 9 & 3 & $<-100$ \\
\hline 20 & -1.6 & 8424 & 6.5 & 3 & 8542 & 276 & 2 & 1.4 & -1 & 2359 & 7.9 & 3 & 675 & 91 & 3 & $<-100$ \\
\hline 21 & -1.3 & 9072 & 4.8 & 3 & 9706 & 185 & 2 & 6.5 & -1 & 1294 & 6.1 & 3 & 1234 & 88.6 & 3 & -4.9 \\
\hline 22 & -10.0 & 7794 & 5.7 & 2 & 6946 & 698 & 2 & -12.2 & 0 & 165 & 4.2 & 3 & 165 & 37.4 & 3 & 0 \\
\hline 23 & 0.0 & 5506 & 14 & 3 & 6290 & 1160 & 2 & 12.5 & -1 & 214 & 3.7 & 3 & 174 & 68.5 & 3 & -23.0 \\
\hline 24 & -7.7 & 7664 & 11.5 & 3 & 7204 & 193.5 & 2 & -6.4 & -1 & 0 & 2.4 & 3 & 0 & 12 & 3 & 0 \\
\hline 25 & - & - & - & - & 9692 & 22.2 & 3 & - & - & - & - & - & 1966 & 40 & 3 & - \\
\hline 26 & - & - & - & - & 7270 & 320 & 3 & - & - & - & - & - & 727 & 32.3 & 3 & - \\
\hline 27 & -6.3 & 7562 & 8.7 & 3 & 7538 & 526.5 & 2 & -0.3 & -1 & 1061 & 11.5 & 3 & 674 & 61.2 & 3 & -57.4 \\
\hline 28 & -10.3 & 8714 & 5.2 & 3 & 7950 & 262 & 2 & -9.6 & -1 & 654 & 4.9 & 3 & 0 & 11.8 & 3 & $<-100$ \\
\hline 29 & -8.2 & 8802 & 3.5 & 3 & 8132 & 18.8 & 2 & -8.2 & -1 & 40 & 4.2 & 3 & 40 & 9.3 & 3 & 0 \\
\hline 30 & 0.0 & 7250 & 62 & 2 & 7250 & 4000 & 2 & 0.0 & 0 & 525 & 2.9 & 3 & 131 & 46.9 & 3 & $<-100$ \\
\hline Average & -5.7 & 7772.3 & 37.9 & 2.6 & 7598.2 & 1262.7 & 2.1 & -3.2 & -0.5 & 571.5 & 7.7 & 3.0 & 274.5 & 62.5 & 3.0 & $<-100$ \\
\hline
\end{tabular}


Table 4

Comparing effect of number of transfers points and the scheme of pickup and delivery system on transfers

\begin{tabular}{|c|c|c|c|c|c|c|c|c|c|c|c|c|c|c|c|}
\hline \multirow{3}{*}{$\begin{array}{c}\text { Problem } \\
\text { No } \\
\end{array}$} & \multirow{3}{*}{$\begin{array}{c}\text { Single transfers point } \\
\text { Triangle scheme } \\
\operatorname{Gap}(\mathrm{z}) \%\end{array}$} & \multicolumn{7}{|c|}{ Two transfers point (Triangle scheme) } & \multicolumn{7}{|c|}{ Single point scheme } \\
\hline & & \multicolumn{3}{|c|}{$\begin{array}{l}\text { I wo transi } \\
\text { PDP }\end{array}$} & \multicolumn{3}{|c|}{ PDPT } & \multirow[t]{2}{*}{$\operatorname{Gap}(z) \%$} & \multicolumn{3}{|c|}{ PDP } & \multicolumn{3}{|c|}{ PDPT } & \multirow[t]{2}{*}{$\operatorname{Gap}(z) \%$} \\
\hline & & $\mathbf{Z}$ & $\mathrm{t}(\mathrm{sec})$ & $\mathbf{v}$ & $\mathbf{z}$ & $\mathrm{t}(\mathrm{sec})$ & $\mathbf{v}$ & & $\mathbf{z}$ & t (sec) & $\mathbf{v}$ & $\mathbf{Z}$ & t (sec) & $\mathbf{v}$ & \\
\hline 1 & 0.0 & 6498 & 2 & 3 & 6498 & 39 & 3 & 0.0 & 7532 & 1.9 & 2 & 7532 & 9.1 & 2 & 0.0 \\
\hline 2 & -13.1 & 8160 & 10.4 & 3 & 7210 & 397 & 3 & -13.2 & 8080 & 6 & 3 & 8080 & 87.4 & 3 & 0.0 \\
\hline 3 & -1.0 & 6864 & 2 & 3 & 6796 & 15.3 & 2 & -1.0 & 7684 & 2.4 & 2 & 7589 & 17.8 & 2 & -1.3 \\
\hline 4 & -5.3 & 8734 & 11.9 & 3 & 8206 & 275 & 3 & -6.4 & 8792 & 8.4 & 3 & 8792 & 108 & 3 & 0.0 \\
\hline 5 & 0.0 & 8224 & 2.5 & 3 & 8224 & 426 & 3 & 0.0 & 8988 & 2.7 & 2 & 8988 & 69.5 & 2 & 0.0 \\
\hline 6 & -11.4 & 7964 & 2.8 & 3 & 7148 & 484 & 3 & -11.4 & 7668 & 2.5 & 3 & 7668 & 19.9 & 3 & 0.0 \\
\hline 7 & 0.0 & 5790 & 5.5 & 2 & 5790 & 100 & 2 & 0.0 & 6806 & 5.9 & 2 & 6806 & 39.9 & 2 & 0.0 \\
\hline 8 & -6.5 & 8386 & 4.4 & 3 & 7756 & 59 & 3 & -8.1 & 9024 & 3.4 & 3 & 8694 & 16 & 2 & -3.8 \\
\hline 9 & -2.7 & 7346 & 2.3 & 2 & 7156 & 14.2 & 3 & -2.7 & 8220 & 2.2 & 2 & 8099 & 13.8 & 2 & -1.5 \\
\hline 10 & - & - & - & - & 8748 & 43.2 & 3 & - & - & - & - & 8960 & 7.3 & 3 & - \\
\hline 11 & -2.4 & 8764 & 1.7 & 3 & 8558 & 32.4 & 3 & -2.4 & 8620 & 2 & 3 & 8620 & 5.3 & 3 & 0.0 \\
\hline 12 & 0.0 & 5552 & 4.3 & 2 & 5384 & 167 & 3 & -3.1 & 6554 & 8.7 & 3 & 6514 & 70.6 & 2 & -0.6 \\
\hline 13 & -8.3 & 8540 & 2.1 & 3 & 7866 & 140 & 3 & -8.6 & 8848 & 2.7 & 3 & 8848 & 107 & 3 & 0.0 \\
\hline 14 & -6.1 & 6600 & 15 & 3 & 6222 & 918 & 3 & -6.1 & 6550 & 8 & 2 & 6312 & 150 & 3 & -3.8 \\
\hline 15 & -17.3 & 9380 & 4.9 & 3 & 7998 & 25.5 & 3 & -17.3 & 9508 & 4.2 & 3 & 9508 & 18.2 & 3 & 0.0 \\
\hline 16 & -0.2 & 6120 & 32.2 & 2 & 6314 & 4000 & 2 & 0.0 & 6246 & 20 & 2 & 6246 & 837 & 2 & 0.0 \\
\hline 17 & -8.2 & 7164 & 10.8 & 3 & 6620 & 261 & 3 & -8.2 & 7498 & 5.2 & 3 & 7272 & 473 & 2 & -3.1 \\
\hline 18 & -10.5 & 7010 & 7.4 & 3 & 6312 & 299 & 2 & -11.1 & 6938 & 2.8 & 3 & 6693 & 13.6 & 3 & -3.7 \\
\hline 19 & -14.3 & 8508 & 6.1 & 3 & 7424 & 68.9 & 3 & -14.6 & 8902 & 3.8 & 3 & 8554 & 23.7 & 3 & -4.1 \\
\hline 20 & -1.6 & 8424 & 2.1 & 3 & 8288 & 8 & 3 & -1.6 & 8582 & 1.8 & 3 & 8582 & 4 & 3 & 0.0 \\
\hline 21 & -1.3 & 9072 & 1.9 & 3 & 8954 & 193 & 3 & -1.3 & 9860 & 2 & 3 & 9860 & 12 & 3 & 0.0 \\
\hline 22 & -10.0 & 7184 & 3.2 & 3 & 6530 & 39.2 & 3 & -10.0 & 7088 & 2.3 & 3 & 7088 & 10.1 & 3 & 0.0 \\
\hline 23 & 0.0 & 5506 & 1.6 & 3 & 5506 & 19.7 & 3 & 0.0 & 6750 & 3.2 & 3 & 6750 & 36.5 & 3 & 0.0 \\
\hline 24 & -7.7 & 7664 & 4.9 & 3 & 7116 & 5.4 & 3 & -7.7 & 8268 & 2.6 & 3 & 8268 & 10.2 & 3 & 0.0 \\
\hline 25 & - & - & - & - & 9022 & 9.2 & 3 & - & - & - & - & 10322 & 2.6 & 3 & - \\
\hline 26 & - & - & - & - & 7270 & 24 & 3 & - & - & - & - & 7836 & 7.3 & 3 & - \\
\hline 27 & -6.3 & 7562 & 4.1 & 3 & 7116 & 14.7 & 3 & -6.3 & 8036 & 2.9 & 3 & 8036 & 12.2 & 3 & 0.0 \\
\hline 28 & -10.3 & 8714 & 3.6 & 3 & 7902 & 95.4 & 3 & -10.3 & 8450 & 2.8 & 3 & 8450 & 44.1 & 3 & 0.0 \\
\hline 29 & -8.2 & 8802 & 1.7 & 3 & 8132 & 6.7 & 2 & -8.2 & 9098 & 1.7 & 3 & 8786 & 2.5 & 2 & -3.6 \\
\hline 30 & 0.0 & 7250 & 4.6 & 2 & 7250 & 325 & 2 & 0.0 & 8142 & 5.8 & 2 & 8142 & 135 & 2 & 0.0 \\
\hline Average & -5.7 & 7621.6 & 5.8 & 2.8 & 7195.4 & 312.2 & 2.8 & -5.9 & 8027.1 & 4.4 & 2.7 & 7954.7 & 86.9 & 2.6 & -0.9 \\
\hline
\end{tabular}


The results of Table 4 show that the addition of the second transfers point increases the average cost reduction from $5.7 \%$ to $5.9 \%$ and the cost reduction is directly related to the number of transfers points. On the other hand, a slight reduction in costs indicates that the location of the transfers point plays a significant role in its effectiveness.

According to the results of the second experiment (changing system scheme), if a single point scheme is used instead of the triangular scheme, the impact rate of the transfers point in cost reduction will be reduced from the average of $5.7 \%$ to $0.9 \%$, which will emphasized on the scheme and layout of the transfers points and depots.

\subsection{Critical condition}

In this section we want to evaluate the benefits of transfers in the critical conditions, including the short or long length of the requests (direct distance between the pickup and delivery points of each request), the short or long time window of requests, and the low or high capacity of vehicles in proportion to the volume of requests. Six experiments were designed for this purpose.

The following experiments were designed based on "initial instances":

1. Limiting the capacity of the vehicles (Single delivery). It is assumed in this experiment, that the vehicle can only carry a single request at the time. Therefore, after pickup, request must be delivered immediately or transferred to a transfer point.

2. Increasing the vehicle's capacity so that it is possible to pick up all requests simultaneously; no capacity limit or high vehicle capacity.

Also, a sample of new problems was created for other experiments. These experiments are designed as follows:

3. Short distance requests. To this end, 30 new problem cases were generated with a random length between 100 to 500 units. Other parameters are the same as the "initial instances".

4. Long distance requests. For this purpose, 30 new problem cases were generated with a random length between 700 to 1,000 units. Other parameters are the same as the "initial instances".

5. Short time windows. To this end, 30 new problem cases were generated with a time window of 1000 time units. Other parameters are the same as the "initial instances".

6. Long time windows. For this purpose, 30 new problems were generated with a time window of 5000 times units. Other parameters are the same as the "initial instances".

The results of these experiments are presented in Table 5. According to these results, with the increase in vehicle capacity, the average utility of the transfers is reduced from $5.7 \%$ in normal condition to $2.3 \%$. On the other hand, transfers benefits are decreased by the decrease in vehicle capacity, but in this case, transfers contribute to a significant increase in system flexibility. System without transfers can process 14 of the 30 cases (47\%); however, this increased to $26(87 \%)$ considering the transfers. The "feasibility ratio" column is obtained by dividing the number of the solvable problems in the PDPT scenario to the number of solvable problems in the PDP scenario, which is 1.86 in this case.

In the case of short window lengths, we have a significant increase in number of solvable problems (feasibility ratio $=1.93$ ) while reducing costs by $4.5 \%$. Increasing flexibility can also be expressed in another way. Suppose that another set of vehicles, other than the three defined vehicles, are available, but the condition for using either of these is the use of the three previous vehicles. By this view, it is possible to eliminate infeasibility, but instead, more cost should be paid for using new vehicles. 
Table 5

Transfers benefits in critical conditions

\begin{tabular}{|c|c|c|c|c|c|c|c|c|c|c|}
\hline \multirow[b]{2}{*}{ Experiment } & \multicolumn{4}{|c|}{ PDP } & \multicolumn{4}{|c|}{ PDPT } & \multirow[b]{2}{*}{$\begin{array}{l}\text { Feasibility } \\
\quad \text { ratio }\end{array}$} & \multirow[b]{2}{*}{$\begin{array}{c}\text { Average Gap } \\
\text { (z) } \%\end{array}$} \\
\hline & $\begin{array}{c}\text { Feasible } \\
\text { count }\end{array}$ & Average $z$ & $\begin{array}{l}\text { Average } t \\
\text { (sec) }\end{array}$ & Average v & $\begin{array}{c}\text { Feasible } \\
\text { count }\end{array}$ & Average $z$ & $\begin{array}{l}\text { Average t } \\
\text { (sec) }\end{array}$ & Average v & & \\
\hline Normal condition & 27 & 7621.6 & 9.4 & 2.8 & 30 & 7204.5 & 83.8 & 2.8 & 1.11 & -5.7 \\
\hline Single delivery & 14 & 9042.4 & 1.9 & 2.9 & 26 & 8661.0 & 4.7 & 2.9 & 1.86 & -4.7 \\
\hline $\begin{array}{l}\text { High vehicle } \\
\text { capacity }\end{array}$ & 30 & 5885.4 & 4.5 & 1.7 & 30 & 5755.7 & 85.1 & 2.0 & 1.00 & -2.3 \\
\hline $\begin{array}{l}\text { Short request } \\
\text { length }\end{array}$ & 28 & 5765.4 & 6.3 & 3 & 30 & 5694.7 & 12.4 & 2.9 & 1.07 & -1.2 \\
\hline $\begin{array}{l}\text { Long request } \\
\text { length }\end{array}$ & 25 & 9651.0 & 8.0 & 2.8 & 30 & 9391.0 & 63.2 & 2.8 & 1.20 & -3.9 \\
\hline $\begin{array}{l}\text { Short time } \\
\text { window }\end{array}$ & 14 & 8419.7 & 4.8 & 2.9 & 27 & 8035.7 & 21.7 & 2.9 & 1.93 & -4.5 \\
\hline Long time window & 30 & 6650.4 & 81.8 & 1.8 & 30 & 6586.8 & 501.9 & 1.9 & 1.00 & -1.1 \\
\hline
\end{tabular}

Table 6

Transfers benefits in heterogeneous conditions

PDPT

Experiment

$$
\text { Feasible count Average } z
$$

Average t (sec)

Average Gap (z) $\%$

\begin{tabular}{|c|c|c|c|c|c|c|c|c|c|}
\hline Normal & 27 & 7621.6 & 9.4 & 2.8 & 30 & 7204.5 & 83.8 & 2.8 & -5.7 \\
\hline Heterogeneous capacity & 18 & 8075.4 & 9.5 & 2.7 & 28 & 7452.2 & 42 & 2.3 & -8.3 \\
\hline Heterogeneous cost rate & 27 & 8939.9 & 8.8 & 2.6 & 30 & 7860.4 & 62.6 & 2.1 & -13.4 \\
\hline
\end{tabular}




\subsection{Heterogeneous vehicles}

Vehicles have been homogenous in terms of capacity and cost rates in the experiments have been conducted so far. However, this is not always the case in the real world. To evaluate the effect of these two parameters on the benefits of transfers, two experiments have been designed and implemented. In the first experiment, we assume that in the "initial instances", vehicles located at points $(250,285)$, $(750,285),(500,715)$ have a capacity of 10,10 , and 5 units, respectively. In the second experiment, we assume that the cost for these three vehicles is 1,1 and 2 , respectively. The results of implementing PDP and PDPT models for this samples are presented in Table 6.

According to the results, transfers plays a positive role in the reduction of costs in both scenarios, through the optimal use of vehicles; so that in the first scenario, the average cost reduction is $8.3 \%$ and in the second scenario, it is $13.4 \%$. In the case of the heterogeneous capacity of the vehicles, the problem without transfers is feasible in 18 cases $(60 \%)$, due to the reduction in the total capacity of the vehicles (25 units versus 30 units); however, considering the transfers, this amount is increased to 28 cases $(93.3 \%)$. In the case of heterogeneous cost rates, the model with transfers has been moved toward using the less expensive vehicles and the average amount of used vehicles decreases from 2.6 to 2.1. We also have a dramatic drop in costs by an average of $13.4 \%$.

\subsection{Vehicle with time window}

In the previous experiments, it is assumed that the vehicle is ready throughout the planning time horizon. In modern transportation systems such as crowdsourcing, the activity of any vehicle has a time window. In this case, the pickup and delivery system without transfers cannot carry out long-distance requests or requests that their route does not completely overlap with a vehicle route.

This problem can be solved by adding the transfers opportunity. Suppose there are six vehicles with a capacity of 10 units and the same cost rate (equal to one unit). Of these, there are three vehicles with a time window from 0 to 5000, respectively, with the origins $(250,285),(750,285)$ and $(500,715)$ and the corresponding destinations $(750,285),(500,715)$ and $(250,285)$ and three vehicles with a time window 5000 to 10000 , respectively with the origins of $(250,285),(750,285)$ and $(500,715)$ and corresponding destinations of $(500,715),(750,285)$ and $(285,750)$. Now consider the "initial instances" with these vehicles (remove default vehicles). The solution to these problems is presented in Table 7. Given the low volume of feasible problems, the average cost in the PDP scenario in this table is not comparable with the corresponding average of the second scenario (PDPT) and is not provided. According to the results, without considering the transfers, it is possible to reach the feasible solution only in 4 cases $(13.3 \%)$, while considering the transfers, it is possible to reach the solution and cover the requests in 28 of the 30 cases $(93.3 \%)$.

\section{Table 7}

Transfers benefits in vehicles with time window case

\begin{tabular}{|c|c|c|c|c|c|c|c|c|c|c|}
\hline \multirow[b]{2}{*}{ Experiment } & \multicolumn{4}{|l|}{ PDP } & \multicolumn{4}{|l|}{ PDPT } & \multirow[b]{2}{*}{$\begin{array}{l}\text { Feasibility } \\
\text { ratio }\end{array}$} & \multirow{2}{*}{$\begin{array}{l}\text { Average } \\
\text { Gap (z) } \\
\%\end{array}$} \\
\hline & $\begin{array}{l}\text { Feasible } \\
\text { count }\end{array}$ & $\begin{array}{l}\text { Average } \\
\mathrm{z}\end{array}$ & $\begin{array}{l}\text { Average } \\
t(\mathrm{sec})\end{array}$ & $\begin{array}{l}\text { Average } \\
\mathbf{v}\end{array}$ & $\begin{array}{l}\text { Feasible } \\
\text { count }\end{array}$ & $\begin{array}{l}\text { Average } \\
\mathrm{Z}\end{array}$ & $\begin{array}{l}\text { Average } \\
t(\mathrm{sec})\end{array}$ & $\begin{array}{l}\text { Average } \\
\mathbf{v}\end{array}$ & & \\
\hline $\begin{array}{l}\text { Vehicle with } \\
\text { time window }\end{array}$ & 4 & - & 13.8 & 4 & 28 & 8776.2 & 926 & 4.2 & 7.0 & -3.7 \\
\hline
\end{tabular}




\section{Discussion and conclusion}

The transfers capability (shifting a load/passenger between two vehicles) is an attractive feature for modern transportation systems. Despite relatively numerous studies in this area, some key issues are remain unsolved. Perhaps the first and most important issue in this regard is whether this feature will be beneficial and to what extent and how it's potential can be exploited. The present study tried to give a fairly comprehensive answer to these questions. According to the results of several experiments, many parameters include the modeling parameters (distance metric and objective function), system design parameters (number and location of the transfers points in the network), operational parameters (number, capacity, time window and cost of vehicles, and length, time window and volume to capacity ratio of requests) have a role in this regard. The significant difference between the system average cost reductions based on Manhattan distance (5.7\%) compared to Euclidean distance (4.2\%), shows that approaching the real distance between network nodes, the transfers capability shows its effect more. It is expected to achieve different, more promising results using the time-dependent distance and removal of the triangular inequality in the infrastructure network. Also, this impact varies depending on the objective function, and it is more significant towards reducing the delay (a $100 \%$ reduction in average latency from 571 to 274 time unit) or the reduction of used vehicles (from an average of 2.6 to 2.1, while $3.2 \%$ decrease in cost function) than reducing the cost of the traveled distance. In the critical conditions, such as equality of the vehicle capacity and quantity of requests (vehicle can only carry a single request at a time) or the short time window of requests, the impact of transfers opportunity on the system's response (the ability to response the requests) is significant (increasing the number of feasible requests from 14 to 26 cases, equivalent to an increase of $46.6 \%$ to $86.6 \%$ in the first case and from 14 to 27 cases, $46.6 \%$ to $90 \%$ in the second case).

In case of heterogeneous vehicles in terms of capacity and cost rates, the use of transfers can reduce costs dramatically (average cost reductions of $8.3 \%$ and $13.4 \%$, respectively in the first and second cases, in the generated samples). On the other hand, transfers enable the use of a vehicle fleet with a limited time window, which otherwise would not be possible (an increase in the sample of solvable problems from 4 to 28 cases $\backslash$ from $13.3 \%$ to $93.3 \%$ ).

Although carried out in small scales, the conducted experiments can easily be generalized to large scales, and, as mentioned in the literature, the usefulness and impact of the transfers increase with the problem size. Also, the synergy of the parameters affecting the transfers is an issue that should be addressed. Besides this, the real-world conditions may have many opportunities and benefits for this capability.

There are still many other questions to be addressed and considered in future research. There is still no definite strategy for the design of transportation systems with transfers. Also, there is still much to do on using this feature effectively in practice, in the real world and dynamic conditions. There are many problems in coordinating the vehicles involved in the transfers process, that the technology development has provided an appropriate basis for their solution. 


\section{References}

Bouros, P., Sacharidis, D., Dalamagas, T., \& Sellis, T. (2011). Dynamic Pickup and Delivery with Transfers. Advances in Spatial and Temporal Databases (pp. 112-129).

Coltin, B., \& Veloso, M. M. (2014). Scheduling for Transfers in Pickup and Delivery Problems with Very Large Neighborhood Search. AAAI, 2250-2256.

Cortés, C. E., Matamala, M., \& Contardo, C. (2010). The pickup and delivery problem with transfers: Formulation and a branch-and-cut solution method. European Journal of Operational Research, 200(3), 711-724.

Godart, A., Manier, H., Bloch, C., \& Manier, M.-A. (2018). MILP for a Variant of Pickup \& Delivery Problem for both Passengers and Goods Transportation. 2018 IEEE International Conference on Systems, Man, and Cybernetics (SMC), 2692-2698. IEEE.

Gørtz, I. L., Nagarajan, V., \& Ravi, R. (2009). Minimum makespan multi-vehicle dial-a-ride. In Algorithms-ESA 2009 (pp. 540-552).

Kerivin, H. L. M., Lacroix, M., Mahjoub, A. R., \& Quilliot, A. (2008). The splittable pickup and delivery problem with reloads. European Journal of Industrial Engineering, 2(2), 112-133.

Li, H., \& Lim, A. (2003). A metaheuristic for the pickup and delivery problem with time windows. International Journal on Artificial Intelligence Tools, 12(02), 173-186.

Lotfi, S., Abdelghany, K., \& Hashemi, H. (2019). Modeling Framework and Decomposition Scheme for On-Demand Mobility Services with Ridesharing and Transfer. Computer-Aided Civil and Infrastructure Engineering, 34(1), 21-37.

Masson, R., Lehuédé, F., \& Péton, O. (2013a). An adaptive large neighborhood search for the pickup and delivery problem with transfers. Transportation Science, 47(3), 344-355.

Masson, R., Lehuédé, F., \& Péton, O. (2013b). Efficient feasibility testing for request insertion in the pickup and delivery problem with transfers. Operations Research Letters, 41(3), 211-215.

Masson, R., Lehuédé, F., \& Péton, O. (2014). The Dial-A-Ride Problem with Transfers. Computers \& Operations Research, 41, 12-23.

Masson, R., Lehuédé, F., Péton, O., \& others. (2011). A tabu search algorithm for the Dial-a-Ride Problem with Transfers. Proceedings of the IESM 2011 Conference.

Mitrović-Minić, S., \& Laporte, G. (2006). The pickup and delivery problem with time windows and transshipment. INFOR: Information Systems and Operational Research, 44(3), 217-227.

Mues, C., \& Pickl, S. (2005). Transshipment and time windows in vehicle routing. 8th International Symposium on Parallel Architectures,Algorithms and Networks, 2005. ISPAN 2005.

Nakao, Y., \& Nagamochi, H. (2008). Worst Case Analysis for Pickup and Delivery Problems with Transfer. IEICE Trans. Fundam. Electron. Commun. Comput. Sci., E91-A(9), 2328-2334.

Qu, Y., \& Bard, J. F. (2012). A GRASP with adaptive large neighborhood search for pickup and delivery problems with transshipment. Computers \& Operations Research, 39(10), 2439-2456.

Rais, A., Alvelos, F., \& Carvalho, M. S. (2014). New mixed integer-programming model for the pickup-and-delivery problem with transshipment. European Journal of Operational Research, 235(3), 530-539.

Sampaio Oliveira, A. H., Savelsbergh, M. W., Veelenturf, L. P., \& van Woensel, T. (2018). The benefits of transfers in crowdsourced pickup-and-delivery systems.

Shang, J. S., \& Cuff, C. K. (1996). Multicriteria pickup and delivery problem with transfer opportunity. Computers \& Industrial Engineering, 30(4), 631-645.

Thangiah, S. R., Fergany, A., \& Awan, S. (2007). Real-time split-delivery pickup and delivery time window problems with transfers. Central European Journal of Operations Research, 15(4), 329349. 
